\title{
Physics of Solar-Like Oscillations
}

\author{
Jørgen Christensen-Dalsgaard \\ Teoretisk Astrofysik Center, Danmarks Grundforskningsfond, and \\ Institut for Fysik og Astronomi, Aarhus Universitet, DK-8000 Aarhus \\ $C$, Denmark
}

\begin{abstract}
The physics of solar and stellar oscillations determine their observable characteristics. I provide a brief overview of the properties of solar-like oscillations, excited by stochastic processes, in other stars. In addition, I consider the current state of investigations of such oscillations, as well as the prospects for an improved understanding of their physics and the properties of the pulsating stars.
\end{abstract}

\section{Introduction}

Oscillations of isolated stars may be caused by two fundamentally different mechanisms: intrinsic instability and stochastic excitation. In the former case, the star acts as a heat engine, converting thermal energy into mechanical energy through a proper phasing of local heating and compression; this is the case, for example, in the classical instability strip populated by the Cepheids, RR Lyrae stars and $\delta$ Scuti stars. In the latter case, the stellar oscillations are intrinsically damped, but excited by other processes, typically turbulent flows; this happens in the Sun where the near-surface convection, with nearly sonic speeds, causes emission of acoustic waves which excite the modes of oscillation. Oscillations excited in this manner by convection may be considered solar-like; as discussed below, they are expected to be present in all stars with vigorous near-surface convection and hence occur in both solar-like stars and stars quite unlike the Sun.

As discussed by Kjeldsen \& Bedding in this volume, solar-like oscillations have been observed in a variety of stars in recent years, including both mainsequence stars and highly evolved giants. Further extensive data are expected in the near future, both from continuing ground-based observations and from several space missions under way. Thus it is important to consider the physical properties of the oscillations, and in particular how the observed characteristics reflect the properties of the stars and may be used to probe these properties.

\section{Basic properties of solar-like oscillations}

Only a very brief discussion of the properties of stellar oscillations can be provided here. For more details, Unno et al. (1989) and Gough (1993) may be consulted, for example. 


\subsection{Mode Properties}

A mode of stellar oscillation is characterized by the degree $\ell$ and azimuthal order $m$ which determine the properties of the mode as a function of co-latitude and longitude, and the radial order $n$ which depends on the variation with distance $r$ to the center. Here $\ell$ determines the total number of nodal lines on the stellar surface, while $m$ determines the number of nodes around the equator. From stellar observations, averaging light over the stellar disk typically suppresses the signal from all but the modes of the lowest degree, with $\ell \leq 3$.

The behavior of a mode in the radial direction is determined by the restoring forces: pressure forces caused by compression and rarefaction, and buoyancy forces caused by horizontal density differences being affected by gravity. These forces can be described in terms of two characteristic frequencies, varying through the star: the acoustic (or Lamb) frequency $S_{\ell}$ and the buoyancy (or Brunt-Väisälä) frequency $N . S_{\ell}$ is determined by $S_{\ell}=\sqrt{\ell(\ell+1)} c / r$, where $c$ is the adiabatic sound speed, $c^{2}=\Gamma_{1} p / \rho$, where $p$ is pressure, $\rho$ is density, and $\Gamma_{1}=(\partial \ln p / \partial \ln \rho)_{\text {ad }}$ is the adiabatic exponent. $N$ is given by

$$
N^{2}=g\left(\frac{1}{\Gamma_{1}} \frac{\mathrm{d} \ln p}{\mathrm{~d} r}-\frac{\mathrm{d} \ln \rho}{\mathrm{d} r}\right)
$$

where $g$ is the gravitational acceleration. In regions where $N^{2}>0, N$ is the frequency of a gas element of small horizontal extent oscillating under the effect of buoyancy. Regions where $N^{2}<0$ are convectively unstable, buoyancy giving rise to accelerating direct motion leading to convective energy transport.

Asymptotic analysis shows that the eigenfunction of a mode oscillates as a function of $r$ if its angular frequency $\omega$ satisfies one of two conditions: either $\omega>S_{\ell}, N$ or $\omega<S_{\ell}, N$. In the first case pressure dominates the restoring force and the mode has the nature of a standing acoustic wave; in the second case restoring is dominated by buoyancy and the mode has the character of a standing gravity wave. The former modes are known as acoustic, or $\mathrm{p}$, modes whereas the latter modes are known as gravity, or g, modes.

The observed modes of solar-like oscillation typically have the character of high-order acoustic modes. These satisfy a simple asymptotic relation for the frequencies, usually written in terms of the cyclic frequency $\nu=\omega / 2 \pi$ :

$$
\nu_{n l} \sim \Delta \nu\left(n+\frac{\ell}{2}+\alpha\right)
$$

to lowest order of approximation, where $\alpha$ is a surface phase. Hence the spectrum is uniformly spaced in order, by the large separation $\Delta_{n \ell}=\nu_{n \ell}-\nu_{n-1 \ell} \sim$ $\Delta \nu \simeq\left(2 \int_{0}^{R} \mathrm{~d} r / c\right)^{-1}$, i.e., the inverse of the sound travel time across a stellar diameter. Also, modes of the same $n+\ell / 2$ are degenerate to this approximation. Corrections to Eq. (2) give rise to a departure from this degeneracy, characterized by the small separation $\delta_{n \ell}=\nu_{n \ell}-\nu_{n-1 \ell+2}$.

In the core of evolved stars the buoyancy frequency attains very high values, due to the strong central condensation and the resulting high value of $g$, often enhanced by a steep gradient in the hydrogen abundance (e.g., outside a convective core). In such cases the buoyancy frequency in the core may exceed 
typical values for the observed frequencies. Here, therefore, a mode can show g-mode-like behavior in the core and p-mode-like behavior in the envelope.

A very useful characteristic of a mode is its mode mass $M_{\text {mode }}$ defined by $M_{\text {mode }}=\int_{V} \rho|\delta \mathbf{r}|^{2} \mathrm{~d} \mathbf{V} /\left|\delta \mathbf{r}_{\mathrm{ph}}\right|^{2}$ where the integration is over the volume $V$ of the star; here $\delta \mathbf{r}$ is the displacement and $\left|\delta \mathbf{r}_{\mathrm{ph}}\right|$ is its surface norm. This relates the surface rms velocity $\left|\mathbf{V}_{\mathrm{ph}}\right|$ to the total energy $\mathcal{E}$ of the mode through $\mathcal{E}=M_{\text {mode }}\left|\mathbf{V}_{\mathrm{ph}}\right|^{2}$. Evidently, modes trapped in the deep interior of a star, such as g modes, tend to have a high value of $M_{\text {mode }}$ one may then suspect that they are less likely to be excited to large amplitudes.

Rotation introduces a dependence of the frequencies on $m$. For slow rotation this rotational splitting is proportional to the rotation rate; this is a good approximation in the solar case. For rapidly rotating stars, however, effects of higher order in the rotation rate must be taken into account, substantially complicating the frequency spectrum (e.g., Soufi et al. 1998).

\subsection{Mode excitation}

As discussed in the introduction, solar-like oscillations are intrinsically damped but excited stochastically by near-surface convection. Thus the mode energies are determined by a balance between the energy input, which typically depends only on the frequency, and the damping rate (e.g., Goldreich et al. 1994; Samadi et al. 2003; see also Stein et al., this volume). Very roughly, the resulting energy is predominantly a function of frequency; at a given frequency the dependence of the surface amplitude on mode properties is then determined by the mode inertia as $\left|\mathbf{V}_{\mathrm{ph}}\right| \propto M_{\text {mode }}^{-1 / 2}$.

Stochastic excitation produces a characteristic frequency variation of the surface amplitude, leading to the largest amplitudes in the solar case near periods of 5 minutes. At low frequencies the modes have low amplitude in the region of efficient excitation, compared with the overall amplitude, leading to small surface amplitudes. Also, driving is most efficient for modes whose periods match the time scale of the near-surface convection, in the solar case 5-10 minutes. At high frequency, at and above the so-called acoustical cut-off frequency, the modes are no longer reflected at the photosphere and suffer substantial energy loss through running waves. For stars that are not too highly evolved this places the largest amplitudes in the frequency range corresponding to high-order acoustic modes, confirming the applicability of the asymptotic Eq. (2).

Christensen-Dalsgaard \& Frandsen (1983) made a very rough estimate of the amplitude expected for stochastically excited modes in a variety of stars, based on an early analysis by Goldreich \& Keeley (1977). Later estimates by Houdek et al. (1999) largely confirmed the earlier results. These estimates are now being tested by data on solar-like oscillations. Interestingly, the observed amplitude for Procyon, which is somewhat hotter than the Sun, is lower by a factor of roughly three than the prediction (e.g., Barban et al. 1999).

\section{Diagnostic potential of solar-like frequencies}

The asymptotic relation in Eq. (2) defines the basic structure of the spectrum of solar-like oscillations and provides the basis for the simplest analysis of observed 
frequencies, in the determination of the large and small frequency separations. Indeed, the regular spacing of the frequency spectrum, together with the characteristic distribution of power with frequency, are the two main signatures of the presence of solar-like oscillations. The large separation provides an average of the entire structure of the star; for stars on or near the main sequence it may be taken as a measure of stellar mass. On the other hand, the small separation is sensitive to the structure of the core of the star; in particular, $\delta_{n \ell}$ is reduced with increasing stellar age. This suggests illustrating the two separations in a two-dimensional diagram, similar to the classical Hertzsprung-Russell diagram (e.g., Christensen-Dalsgaard 1984, 1988; Ulrich 1986). Assuming that other parameters of a star are known, such as its composition, the observed location of the star in this diagram determines its mass and evolutionary state (but see also Gough 1987).

Although the small separation is determined principally by conditions in the core, it retains some sensitivity to overall structure, including detailed properties of the stellar envelope. Roxburgh \& Vorontsov (2003) showed that ratios such as $r_{n l}=\delta_{n \ell} / \Delta_{n \ell}$ are entirely determined by the core conditions and hence provide a much cleaner probe of the age of the star or other aspects of its core structure.

The oscillation frequencies evidently contain information beyond the large and small separations. In particular, the asymptotic description assumes a smooth model, on the scale of the eigenfunction variations. Sharp features give rise to detectable oscillatory signals in the frequencies. In the solar case this signal has been used to investigate the properties of the base of the convective envelope, where the sudden change from adiabatic to sub-adiabatic temperature gradient causes a similarly sharp change in the gradient of the sound speed (e.g., Basu et al. 1994; Monteiro et al. 1994; Roxburgh \& Vorontsov 1994). Also, the decrease of $\Gamma_{1}$ in the region of second helium ionization causes a localized change in the sound speed, with a detectable signature in the frequencies which provides a measure of the helium content of the solar envelope (e.g., Vorontsov et al. 1991; Antia \& Basu 1994; Pérez Hernández \& Christensen-Dalsgaard 1994). Similar analysis may become possible in the stellar case, once data of sufficient quality have been obtained (e.g., Lopes et al. 1997; Pérez Hernández \& ChristensenDalsgaard 1998; Monteiro et al. 2000; Miglio et al. 2003).

\section{Asteroseismology of evolved stars}

After the star leaves the main sequence, following the exhaustion of hydrogen at the center, the buoyancy frequency in the deep interior increases strongly as a result of the contraction of the core. As discussed above, modes in the observed range of frequency may then have a dual character, with p-mode properties in the envelope and g-mode properties in the interior. In most cases the mode is dominated by one of the two possible regions of oscillatory behavior; if this is the g-mode region the resulting mode mass is typically so high that the mode is unlikely to be observed. However, some modes can have substantial amplitudes in both regions; this results in a modest mode mass, and hence a potentially detectable amplitude, while the g-mode behavior causes significant departures from the simple asymptotic relation, Eq. (2). An example of such behavior 
may be present in the sub-giant $\eta$ Bootis which was found by Kjeldsen et al. (1995) to show solar-like oscillations; computed frequencies (e.g., ChristensenDalsgaard et al. 1995; Guenther \& Demarque 1996; Di Mauro et al. 2003) show clear departures from Eq. (2) for $\ell=1$, at mode masses indicating that the modes might be observable. Interestingly, there is some evidence that the observed frequencies show similar behavior; this would be a first indication that properties of the buoyancy frequency can be probed with solar-like oscillations.

The estimates of mode amplitudes indicate that larger amplitude may be expected in more evolved stars, particularly for red giants. Some indications for solar-like oscillations were found in the K0 giant $\alpha \mathrm{UMa}$ from observations made with the WIRE satellite (Buzasi et al. 2000) although the interpretation of the data was later questioned (Dziembowski et al. 2001). Frandsen et al. (2002) found clear evidence for solar-like oscillations in the G7 giant $\xi$ Hya, with an envelope of power strongly reminiscent of the solar case, albeit with an amplitude maximum at a period of around 3 hours. This star is probably in the core helium burning stage, with an extremely condensed core. It is likely, as discussed by Dziembowski et al. (2001), that the resulting very rapid variation of the eigenfunctions in the region of g-mode behavior causes strong damping of non-radial modes, such that only modes with $\ell=0$ are excited to observable amplitudes. Indeed, the observed uniform spacing of the frequencies corresponds to the full large separation $\Delta \nu$ for the most plausible models, rather than half this value as would be observed if both $\ell=0$ and 1 modes were observed.

For even more luminous red giants the predicted amplitudes are quite substantial but the periods are very long, of order weeks or months, making observations extremely time consuming. For the extreme case of asymptotic giant branch stars literally decades of observations are required to identify the frequency structure and other properties of the oscillations. It was argued by Christensen-Dalsgaard et al. (2001) that data on semi-regular variables, obtained through very extensive efforts by the American Association of Variable Star Observers showed statistical properties consistent with stochastic excitation of a solar-like nature. Thus it seems plausible that solar-like oscillations can be observed throughout the red part of the Hertzsprung-Russell diagram, extending to quite extreme cases very different from the Sun. The full diagnostic potential of these data still needs to be explored, however.

Acknowledgments. This work was supported in part by the Danish National Research Foundation through its establishment of the Theoretical Astrophysics Center.

\section{References}

Antia, H. M., \& Basu, S. 1994, ApJ, 426, 801

Barban, C., Michel, E., Martic, M., Schmitt, J., Lebrun, J. C., Baglin, A., \& Bertaux, J. L. 1999, A\&A, 350, 617

Basu, S., Antia, H. M., \& Narasimha, D. 1994, MNRAS, 267, 209

Buzasi, D., Catanzarite, J., Laher, R., Conrow, T., Shupe, D., Gautier III, T. N., Kreidl, T., \& Everett, D. 2000, ApJ, 532, L133

Christensen-Dalsgaard, J. 1984, in Space Research Prospects in Stellar Activity and Variability, eds A. Mangeney \& F. Praderie (Paris: Paris Observatory Press), 11 
Christensen-Dalsgaard, J. 1988, in IAU Symp. 123, Advances in helio- and asteroseismology, eds J. Christensen-Dalsgaard \& S. Frandsen (Dordrecht: Reidel), 295

Christensen-Dalsgaard, J., \& Frandsen, S. 1983, Solar Phys., 82, 469

Christensen-Dalsgaard, J., Bedding, T. R., \& Kjeldsen, H. 1995, ApJ, 443, L29

Christensen-Dalsgaard, J., Kjeldsen, H., \& Mattei, J. A. 2001, ApJ, 562, L141

Di Mauro, M. P., Christensen-Dalsgaard, J., Kjeldsen, H., Bedding, T. R., \& Paternò, L. 2003, A\&A, 404, 341

Dziembowski, W. A., Gough, D. O., Houdek, G., \& Sienkiewicz, R. 2001, MNRAS, 328, 601

Frandsen, S., Carrier, F., Aerts, C., et al. 2002, A\&A, 394, L5

Goldreich, P., \& Keeley, D. A. 1977, ApJ, 212, 243

Goldreich, P., Murray, N., \& Kumar, P. 1994, ApJ, 424, 466

Gough, D. O. 1987, Nature, 326, 257

Gough, D. O. 1993, in Astrophysical fluid dynamics, Les Houches Session XLVII, eds J.-P. Zahn \& J. Zinn-Justin (Amsterdam: Elsevier), 399

Guenther, D. B., \& Demarque, P. 1996, ApJ, 456, 798

Houdek, G., Balmforth, N. J., Christensen-Dalsgaard, J., \& Gough, D. O. 1999, A\&A, 351,582

Kjeldsen, H., Bedding, T. R., Viskum, M., \& Frandsen, S. 1995, AJ, 109, 1313

Lopes, I., Turck-Chièze, S., Michel, E., \& Goupil, M.-J. 1997, ApJ, 480, 794

Miglio, A., Christensen-Dalsgaard, J., Di Mauro, M. P., Monteiro, M. J. P. F. G., \& Thompson, M. J. 2003, in Asteroseismology across the HR diagram, eds M. J. Thompson, M. S. Cunha \& M. J. P. F. G. Monteiro, (Dordrecht: Kluwer), 537

Monteiro, M. J. P. F. G., Christensen-Dalsgaard, J., \& Thompson, M. J. 1994, A\&A, 283,247

Monteiro, M. J. P. F. G., Christensen-Dalsgaard, J., \& Thompson, M. J. 2000, MNRAS, 316,165

Pérez Hernández, F., \& Christensen-Dalsgaard, J. 1994, MNRAS, 269, 475

Pérez Hernández, F., \& Christensen-Dalsgaard, J. 1998, MNRAS, 295, 344

Roxburgh, I. W., \& Vorontsov, S. V. 1994, MNRAS, 268, 880

Roxburgh, I. W., \& Vorontsov, S. V. 2003, A\&A, 411, 215.

Samadi, R., Nordlund, A, Stein, R. F., Goupil, M. J., \& Roxburgh, I. 2003, A\&A, 403, 303

Soufi, F., Goupil, M. J., \& Dziembowski, W. A. 1998, A\&A, 334, 911

Ulrich, R. K. 1986, ApJ, 306, L37

Unno, W., Osaki, Y., Ando, H., Saio, H., \& Shibahashi, H. 1989, Nonradial Oscillations of Stars, 2nd Edition (Tokyo: University of Tokyo Press)

Vorontsov, S. V., Baturin, V. A., \& Pamyatnykh, A. A. 1991, Nature, 349, 49 